\title{
Über den Zahnwechsel des Yesso Braunbären, Ursus arctos yesoensis Lyd.
}

\author{
Von \\ Tetsuo Inukai und Kôzô Mukasa \\ Aus dem Zoologischen Institut der Hokkaido Kaiserlichen \\ Universität, Sapporo.
}

Mit 1 Textfigur und 8 Figuren auf Tafel XI.

\section{Einleitung.}

Trotz umfangreicher Ergebnisse von Untersuchungen über den Zahnwechsel mancher Säugetiere wissen wir über denjenigen beim Bären noch recht wenig. Schlosser ('09), Kormos ('16), Ehreuberg ('22), Mottl ('34) u. a., die sich mit den Höhlenbären beschäftigten, haben dieses Thema nur anhangsweise berührt; Pohle ('23) war der erste, der eigentlich über die Gebissentwicklung des Bären gearbeitet hat, wobei er hauptsächlich als Material Eisbären benutzte, die er aber durch 2 junge Braunbären und einen Bastard zwischen beiden eben genannten ergänzte.

Zum Glück kommen bei uns in einem ganz eng begrenzten Gebiet, der Insel Hokkaido, noch oft einheimische braune Wildbären vor, die Ursus arctos yesoensis benamnt werden. Neuerdings haben wir eine grosse Anzahl Schädel dieser Bären bekommen, die einen. Versuch in Bezug auf die Altersangaben zulassen können. Im Vorliegenden möchten wir zunächst das Resultat der Untersuchung über den Zahnwechsel veröffentlichen*. Hierfür standen 26 Exemplare der Schädel als fast lückenloses

\footnotetext{
* Mit Unterstützung der Stiftung von ,Nihongakuzitsushinkokai' haben wir die Untersuchung vorgenommen, und dafür möchten wir unseren herzlichen Dank aussprechen.
} 
Untersuchungsmaterial zur Verfügung, und dazu dienten noch vier lebende junge Tiere im naturgeschichtlichen Museum der Universität Sapporo zur Beobachtung.

\section{Beobachtung.}

Stadium 1. Das jüngste Stadium, das wir beobachtet haben, repräsentiert ein im Museum befindliches Ausstopfungsspezimen (Nr. 38) von etwa $30 \mathrm{~cm}$ Körperlänge, das wahrscheinlich ein 6 Monate alter Foetus sein mag. Das Gebiss dieses Tieres zeigt natürlich die Milchzähne, die mit ihren kleinen Spitzen aus dem Zahnfleisch hervorragen; sie sind Id1 und $\overline{\mathrm{Pd} 2}$; d. h. die zwei ersten oberen Milchschneidezähne (incisivi decidi) und die zwei unteren zweiten Milchpraemolaren. Dazu findet man Pd3-Anlage an der Wucherung der Juga alveolaria.

Stadium 2. Das Milchgebiss ist vollständig entwickelt bei dem nächst älteren Tiere ( $\mathrm{Nr} .39$ von etwa $55 \mathrm{~cm}$ Körperlänge), das etwa einen Monat nach der Geburt gestorben sein mag. Die allgemeine Gebissformel stellt sich, ebenso wie bei den anderen Bärenarten, wie folgt dar:

$$
\text { Id } \frac{1 \cdot 2 \cdot 3}{1 \cdot 2 \cdot 3} \mathrm{Cd} \frac{1}{1} \mathrm{Pd} \frac{1 \cdot 2 \cdot 3}{1 \cdot 2 \cdot 3}
$$

Es sind also 28 Zähne in ganzen. Dabei vertreten Pd1 eigentlich Pd2 des idealen Gebisses des Säugetieres, obgleich echte Pd1 beim Bären keineswegs zur Erscheinung kommen. Der Zwischenraum aller Zähne ist sehr weit; indessen liegt das ganze Gebiss auf die vordere Spitzgegend des Kiefers beschränkt.

Stadium 3. Die Reihenfolge des Wechselsrorganges der Milchzähne ist etwas verschieden bei den einzelnen Individuen. Die Beobachtung bei den lebenden jungen Bärchen ergab, dass die Milchzähne leicht zerbrechlich sind, und daher werden sogar fast alle Zähne unter ungünstigen Bedingungen, z. B. bei der Gefangenhaltung, bald nach der Geburt verschwinden. Jedenfalls sind meistens die Cd die letzten, die ausfallen, wenn auch manchmal ausnahmsweise die Milchpraemolaren am längsten bleiben. Was die Reihenfolge im Erscheinen der Ersatzzähne betrifft, so kommen zuerst die ersten Eckzähne zum Vorschein, und dann die ersten Molaren. Ein Exemplar, das etwa 1 Monat alt ist, zeigt die 'hahnformel wie folgt:

$$
\text { Id } \frac{1 \cdot 2 \cdot 3}{1 \cdot 2 \cdot 3} \mathrm{Cd} \frac{1}{1} \mathrm{Pd} \frac{\frac{1 \cdot 2 \cdot 3}{1 \cdot 2 \cdot 3}}{\mathrm{P} 1}
$$


während andere M1 statt P1 aufweisen. Die P1 brechen zwischen Cd und Pd1 durch, und M1 gerade caudal von Pd3.

Ein auch lebender 4 monatiger Bär besitzt neu erschienene M2, P4 und I1.

Stadium 4. Das vierte Stadium ist dargeboten durch einen macerierten Schädel, der etwa 5 Monate alt ist, mit Profillänge von $190 \mathrm{~mm}$ (Nr. 60. Fig. 1). Bei diesem Fall besteht das Gebiss aus verschiedenartigen Zähnen, da schon einige Dauerzähne entwickelt sind, während manche Milchzähne ausgefallen sind. Im Oberkiefer sind 7 Milchzähne Id1, Id2, $\underline{\operatorname{Pd} 2}$ und $\operatorname{Pd} 3$ verschwunden, und im Unterkiefer sind 9, nämlich $\overline{\mathrm{Id} 1}, \overline{\mathrm{Id} 2}, \overline{\mathrm{Pd} 1}, \overline{\mathrm{Pd} 2}$ und $\overline{\mathrm{Pd} 3}$ auch weggegangen. Also bleiben noch als Milchzähne $\mid \mathrm{Id} 2, \mathrm{Id} 3, \mathrm{Cd}, \mathrm{Pd} 1, \overline{\mathrm{Id} 3}, \overline{\mathrm{Cd}}$ und $\overline{\mathrm{Pd} 1}$ ungewechselt, die aber meistens degenerative Neigung zeigen, indem die Wurzeln von $\mathrm{Id} 2, \underline{I d} 3, \underline{C d}$ und $\overline{\mathrm{Id} 3}$ besonders resorbiert sind. Die starken Ersatzzähne $11, \overline{\mathrm{P}} 4, \mathrm{M} 1, \overline{\mathrm{I} 1}, \overline{\mathrm{I} 2}, \overline{\mathrm{P} 1}, \overline{\mathrm{P} 4}$ und $\overline{\mathrm{M} 1}$ sind in Alveolen, die alle um die Wurzeln bereits fertig geschlossen sind. I3 und 1 I2 haben gerade mit der Spitze den Kiefer durchbrochen, und der linke 12 steht bis zum Hals hervor. Die C sind nur äusserlich an der Aufwölbung des Maxillare zu erkemnen. Die P1 sind noch nicht vollständig ausgebildet, sondern ihre Alveolen stehen noch eng offen, und die P3 liegen noch tief in Kiefer. Der vordere Teil der $\overline{M 2}$ ist zwar schon über den Kieferrand gestiegen, aber die M2 und $\overline{\mathrm{M} 3}$ sitzen mit ihrem ganzen Umriss unter der Haut im Alveolenrande. Wir können also das Gebiss dieses Tieres graphisch darstellen, wie folgt:

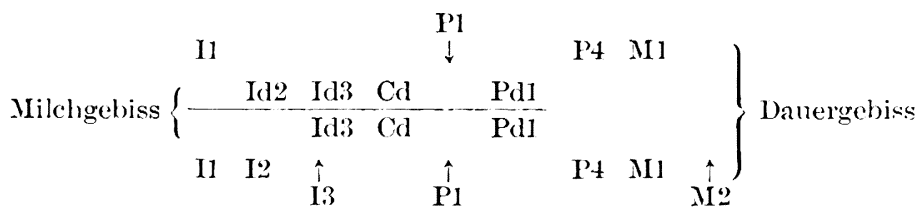

Die Stellung der Dauerzähne ist natürlich charakteristisch für den einzelnen Zahn. Im oberen $Z$ wischenkiefer, finden wir die länglichen ovalförmigen Alveolen für die I1, die schon fertig geschlossen sind, und die noch offenen ovalförmigen der I2 und I3. Die Milchzähne Id1 und $\underline{\text { Id} 2}$ befinden sich am labialen Teil des Lochs, und Id3 sitzen auch labial am cranialen Ende. In Maxillare sehen wir die grossen elliptischen gemeinsamen Alveolen für die $\underline{\mathrm{C}}$ und $\underline{\mathrm{Cd}}$, deren letztere sich am caudalen Ende der Alveole befinden, getrennt von ersteren durch eine unmerkbar geringe Scheidewand. Gerade hinter dem $\mathrm{Cd}$ liegt die länglich ovale Alveole des $\underline{P 1}$, und dann kommt die ganz kleine schräge Alveole für 
den Pd1. Direkt vorn von der Wurzel des P4, lingual, findet man ein kleines Durchbruchsloch des P3 und labial eins für den degenerierenden $\underline{\mathrm{Pd}}$. Die grosse trapezoidische $\mathrm{M} 1$-Alveole liegt posterior von $\mathrm{P4}$. Noch weiter kaudal liegt das Durchbruchsloch für den M2, das eine lange Spalte darstellt. Drei Viertel der Länge des M2 liegen unter der Augenhöhle zum grossen Teil noch verdeckt von der dümmen Knochenlamelle.

Am schmalen Ende des Unterkiefers, und zwar am seiner labialen Seite, sieht man keilförmige Alveolen für die $\overline{\mathrm{I}}$ und etwas seitlich dahinter länglich elliptische, eng offene Alveolen von $\overline{12}$. Noch weiter seitlich, etwas melır nach vorn, liegen die grossen ovalen Löcher für die $\overline{\mathrm{I} 3}$ und $\overline{\mathrm{Id} 3}$; die letzteren Zähne, die degeneriert sind, befinden sich im Gegensatz zu den entsprechenden im Oberkiefer an der caudalen Seite der Löcher während an der gegenüberliegenden vorderen Seite die Spitzen der vorderen Zähne durchbrechen. Keine klares Septum ist zwischen $\overline{\mathrm{I} 2}$ und $\overline{\mathrm{I} 3}$. Auf diese folgen die dreieckigen gemeinsamen Alveolen der $\overline{\mathrm{Cd}}$ und $\overline{\mathrm{C}}$, die von denjenige der $\overline{\mathrm{I}}$ durch die dünne Knochenwand getrennt sind. Die $\overline{\mathrm{Cd}}$ liegen am äusseren labialen Rand des Lochs, während $\overline{\mathrm{C}}$-Anlage lingual neben den ersteren $z u$ finden ist. Caudal von den $\overline{\mathrm{Cd}}$ sehen wir ovale Alveolen für $\overline{\mathrm{P} 1}$, und noch weiter caudal liegen die kleinen, runden Alveolen der $\overline{\mathrm{Pd} 1}$, von denen die linke nur eine Spur zeigt, da sie, besonders auf der linken Seite, schon annähernd geschlossen sind. Die Alveole für dem $\overline{\mathrm{M} 2}$ ist verbunden mit derjenigen für den $\overline{13}$, ohne klare Scheidewand.

Stadium 5. Das nächste Stadium ist durch ein etwa von 6 Monate altes Tier (Nr. 74. Fig. 2) mit Profillänge $206 \mathrm{~mm}$ vertreten. Das ganze Gebiss ist graphisch darstellt wie folgt:

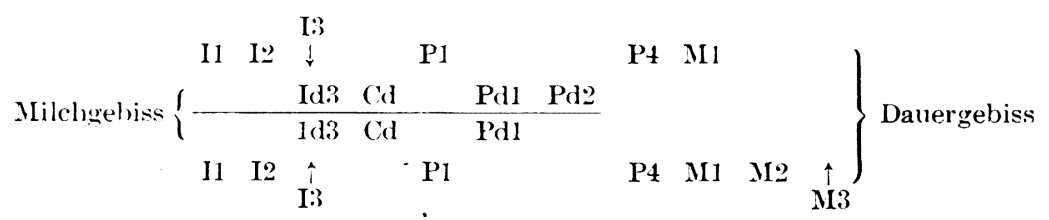

Wir sehen, dass Id3, Cd und $\operatorname{Pd} 2$ von den Milchzähnen noch vorhanden sind, während Id2 kürzlich verschwunden sind. Neue Dauerzähne I2 und I3 kommen zur Erscheinung an Stelle von Id2 bzw. Id3. $\overline{\mathrm{I} 3}$ und $\overline{\mathrm{I2}}$, die beim früheren Stadium sehr klein waren, sind etwas auffallend gewachsen. Die Anlagen von C, P3, und M2 sind noch tief im Kiefer versteckt. Die Alveole des $\overline{\mathrm{Pd} 2}$ ist mit dem Durchbruchsloch des $\underline{\mathrm{P} 3}$, der noch nicht erschienen ist, zu einem gemeinsamen Loch verschmolzen; dabei sitzt $\underline{\operatorname{Pd} 2}$ am labialen Teil dieses Lochs wie 
Über den Zahnwechsel des Yesso Braunbären, Ursus arctos yesoensis Lyd.

im vorigen Stadium. Die länglichen Löcher für den Durchbruch der M2 und $\overline{\mathrm{M} 3}$ sind bei diesem Fall vergrössert, besonders in der Breite. $\overline{\text { Das }}$ vordere Drittel der $\overline{\mathrm{M} 3}$ ist durch das Loch erschienen.

Stadium 6. Das nächste Stadium ist bei einem etwa 7 Monate alten Tier mit Profillänge $160 \mathrm{~mm}$ (Nr. 80. Fig. 3) zu sehen, dessen Gebiss graphisch wie unten gezeigt wird:

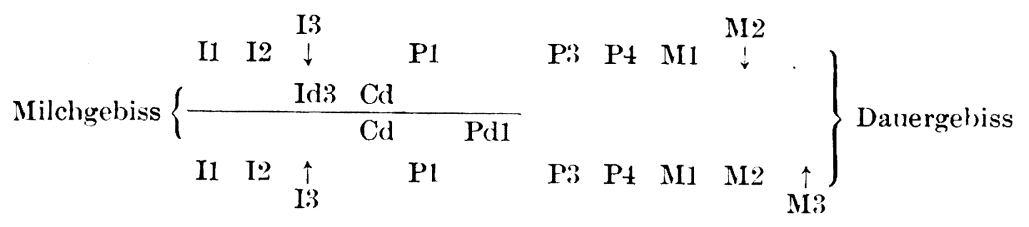

Weiter sind die $\underline{\mathrm{Pd} 1}$ und $\overline{\mathrm{Id} 3}$ aus dem Milchgebiss verloren gegangen, und es gibt von demselben nur noch Id3, Cd und $\overline{\mathrm{Pd} 1}$, deren Wurzeln aber stark resorbiert sind. Die Kronen der I3, die beim vorhergehenden Exemplar nicht vollendet ausgebildet waren, haben schon die Kieferhöhe erreicht, und die $\overline{\mathrm{I} 3}$ sind bis zum Hals durchgebrochen. Dazu sind P3 sehr deutlich geworden. Das Durchbruchsloch für $\underline{\text { M2 }}$ ist in Oberkiefer ziemlich vergrössert.

Stadium 7. Als das nächst ältere Stadium dienen 2 Schädel im Alter von \& Monaten; (Nr. 48. Fig. 4. und Nr. 75) mit Profillänge 188 resp. $204 \mathrm{~mm}$. Die unten gegebene Formel stellt das Gebiss dar :

(Nr. 48)

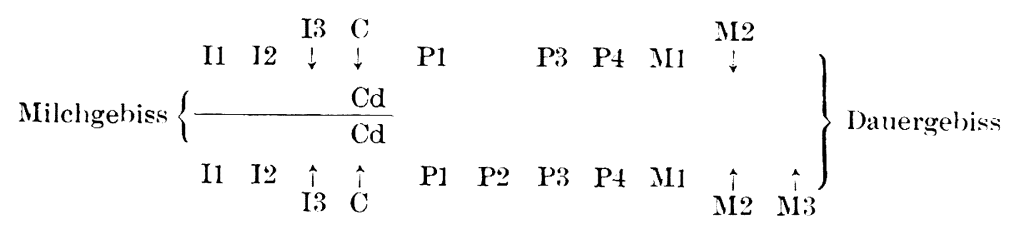

Der $\mathrm{Id} 3$ ist eben verschwunden, und es gibt eine fast unsichtbare Spur des $\overline{\mathrm{Pd}} 1$. Also sind an Milchzähnen nur die Cd vorhanden. Die gerade eben zum Vorschein gekommenen $\mathrm{C}$ haben noch nicht ihre eigentliche Form gewonnen. Bei $\mathrm{Nr} .75$ sind die I3 bis zum Hals emporgekommen und die $\overline{\mathrm{M} 3}$ mit den vergrösserten Durchbruchslöchern auf dem Weg der Erscheinung. Die Alveolen der I1, I2, P1, P3, P4 und M1 sind schon fast vollständig geschlossen. Die $\underline{\mathrm{M} 2}$ befinden sich in sehr schräger, hinten nach oben gerichteter Stellung, so dass nur das vordere Drittel aus dem Zahnfleisch herausragend erscheint. Diese schräge Stellung gilt übrigens für die letzten oberen Kauzähne bei allen Bären, wie Schäff ('89) erwähnt hat. Man findet die $\overline{\mathrm{P} 2}$ und den linken $\overline{\mathrm{P} 3}$ 
bei Nr. 48. Nr. 75 hat noch Pd1 erhalten. Fast im ganzen Umriss sind hier auch die $\overline{\mathrm{M} 3}$ sichtbar, die auch schräg liegen, entsprechend den M2.

Stadium 8. Das nächste Stadium wird auch durch 2 Schädel vertreten, die etwa 10 Monate alt geschätzt werden (Nr. 2 Fig. 5 und Nr. 97), mit Profillänge von je 191 und $215 \mathrm{~mm}$. Das Gebiss verhält sich wie beim vorigen Stadium, abgesehen davon, dass die Wurzeln der Cd mit Ausnalnme der vom rechten $\overline{\mathrm{Cd}}$ bei Nr. 97, die noch stark ist, schon zum grossen Teil resorbiert sind. Nr. 2 hat noch Pd1, aber es fehlt P1; Nr. 97 besitzt die Spur der $\overline{\mathrm{Pd} 1}$.

Stadium 9. Bei den etwa 11 Monate alten Tieren (Nr. 98, 44, 3 und 90 mit Profillängen von 219, 202, 227 und $222 \mathrm{~mm}$ ) ist die Gebissformel dieselbe wie beim eben geschilderten Stadium. Fig. 6 zeigt das Gebiss von Nr. 98. Die Wurzeln der Cd sind zum grossen Teil resorbiert oder abgebrochen. Die I3 sind bei Nr. 90 und ausserdern bei Nr. 3 auch die M2 beinahe vollständig ausgebildet, während die $\overline{\mathrm{M} 3}$ noch auf dem Wege der Steigung sind, mit ihrem vorderen Teil bis zum Hals emporragend. Bei Nr. 9o haben die C mit ihrer Spitze den Kiefer gerade durchbrochen. Man findet bei Nr. 44 die Spur des rechten $\overline{\operatorname{Pd} 1}$ und linken $\overline{\mathrm{Pd} 2}$ und bei $\mathrm{Nr} .90$ die des linken $\mathrm{Pd} 1$ und $\overline{\mathrm{Pd} 1}$. Alle anderen bleibenden Zähne sind viel stärker geworden.

Stadium 10. Im weiteren Verlauf der Entwicklung der Ersatzzähne, nämlich bei Bären im Alter von etwa 12 Monaten (Nr. 99, 12, 61, 11, 103, 49, 101 mit Profillängen von 290, 230, 218, 229, 234, 246 und $253 \mathrm{~mm}$ ) sind alle Milchzähne, mit Ausnahme von nur kleinen Scherbchen der abgebrochenen Wurzeln der $\overline{\mathrm{Cd}}$ bei $\mathrm{Nr}$. 99, verschwunden. Die C sind noch nicht vollständig herausgekommen, und nur bei $\mathrm{Nr} .103$ sind noch die I3 eben bis zum Hals durchgebrochen. Die Alveolen der C sind ziemlich enger geworden. Die M3 sind nur bei $\mathrm{Nr}$. 99 schon ausgebildet. Es gibt noch Pd1 bei Nr. 99, den linken Pd1 bei Nr. 61 und $\overline{\mathrm{Pd} 2}$ bei Nr. 103, während $\mathrm{Nr} .11 \overline{\mathrm{P} 3}, \mathrm{Nr} .49 \mathrm{P} 2$ und $\overline{\mathrm{P} 2}$, und Nr. 101 $\mathrm{P} 2$ und linke $\overline{\mathrm{P} 2}$ haben. Fig. 7 stellt das Gebiss von $\mathrm{Nr} .99$ dar.

Stadium 11. Das älteste Stadium, über das wir gehandelt haben, findet sich bei etwa 13-17 Monate alten Tieren (Nr. 65, 72, 86, 102= Geschwister von $\mathrm{Nr} .103,34,14,13,22,27$, mit Profillänge resp. 260, 224, 239, 253, 275, 275, - 290 und $294 \mathrm{~mm}$ ). Die ( sind bei Nr. 65 (Fig. 8) etwa zu 2/3 durchgebrochen, aber bei Nr. 27 sind sie vollständig herausgekommen. Die letzten Molaren bei $\mathrm{Nr} .27$ sind ausgewachsen ebenso stark wie beim erwachsenen Tier. Der Zahnwechsel ist in diesem Stadium vollendet. Bei Nr. 65 sind die Praemolaren, P1, P2, P3 und 
P4 im Oberkiefer and P1 und P4 im Unterkiefer entwickelt. Die Erscheinung der Praemolaren ist indessen nicht bei allen Individuen dieselbe, sondern zeigt ziemlich grosse Abweichungen, wie wir es schon manchmal in vorhergehenden Stadien bemerkt haben. Nur P4 befinden sich dagegen ausnahmlos bei allen Fällen.

\section{Zusammenfassung.}

Fassen wir nun die Beobachtungen kurz zusammen, so ergibt sich die Reihenfolge des Ausfalls der Milchzähne und der Erscheinung der Dauerzähne wie in folgender Textfigur gezeigt :

Textfigur 1 .

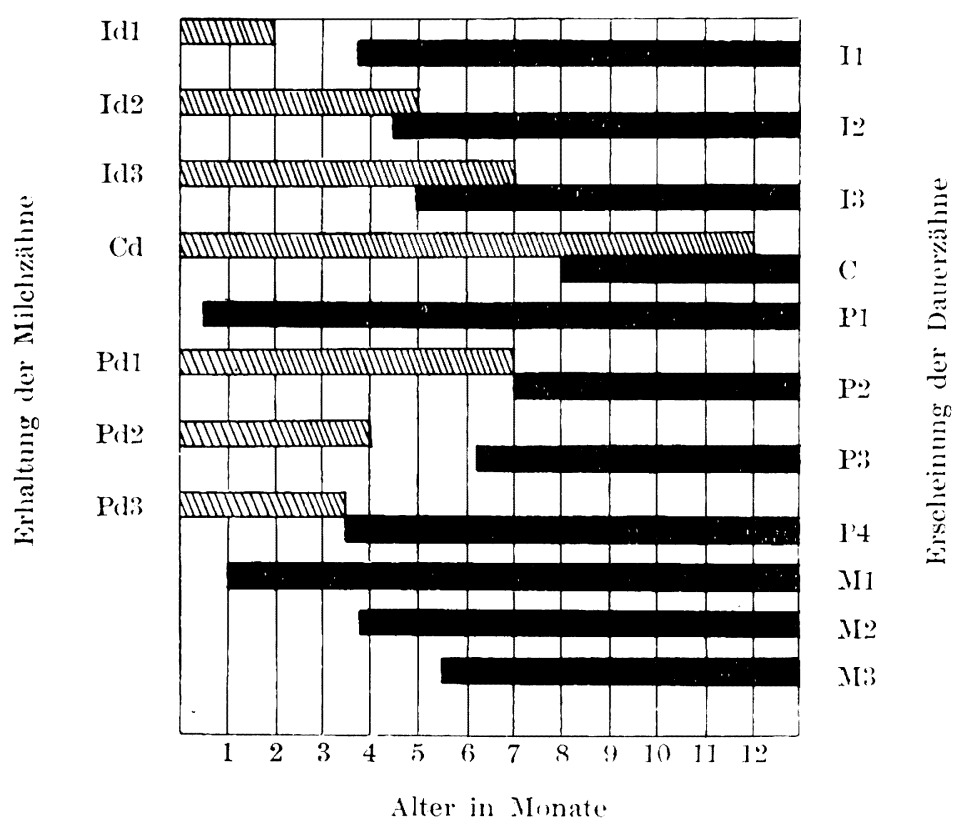

Pohle ('23) hat sich mit den Schädeln von einem 3 Monate, einem 4 Monate alten Braunbären, einem $5 \frac{1}{2}$ monatigen Bastardbären von Eisund Braunbären und von einer Anzahl älterer Eisbären beschäftigt und hat alle Bärenarten bezüglich des Zahnwechsels für übereinstimmend gehalten. Nach ihm sind die Alveolen der (d beim Eisbären von den Durchbruchslöchern der Dauer-C klar getrennt, während sie bei unserem Bären annähernd gemeinsam verschmolzen sind. Übrigens erscheint der Zahnwechsel etwas früher bei den Yesso-Braunbären als bei den 
Eishären, da Id3 bei den ersteren schon nach 7 Monaten und Cd nach 12 Monaten ausfallen während sie bei den letzteren erst nach 13 Monaten resp. 19 Monaten weggehen.

Ausserdem werden die $\mathrm{C}$ des Eisbären in 16-24 Monaten fertig ausgebildet, während dieselben bei dem Yesso-Braunbären schon in 13-17 Monate beinahe vollständig sind. Bei dem sibirische Braunbären brechen nach Middendorff ('88) die I3 und die C gleichzeitig durch, aber bei unserem Hokkaido-Bären kommen I3 viel früher zum Vorschein als C.

Was den Praemolar unseres Bären betrifft, so besteht zwar ziemlich grosse Variabilität bezüglich der Häufigkeit der Erscheinung wie schon gezeigt ist, aber darauf kommen wir bei weiterer Gelegenheit ausführlich zurück. Es werden daher augenblicklich für das Verständnis nur einige Schlussbemerkungen genügen, wenn man die folgende Tabelle betracht :

Nr. der Tiere | Alter in Monaten | vorhandene Praemolaren

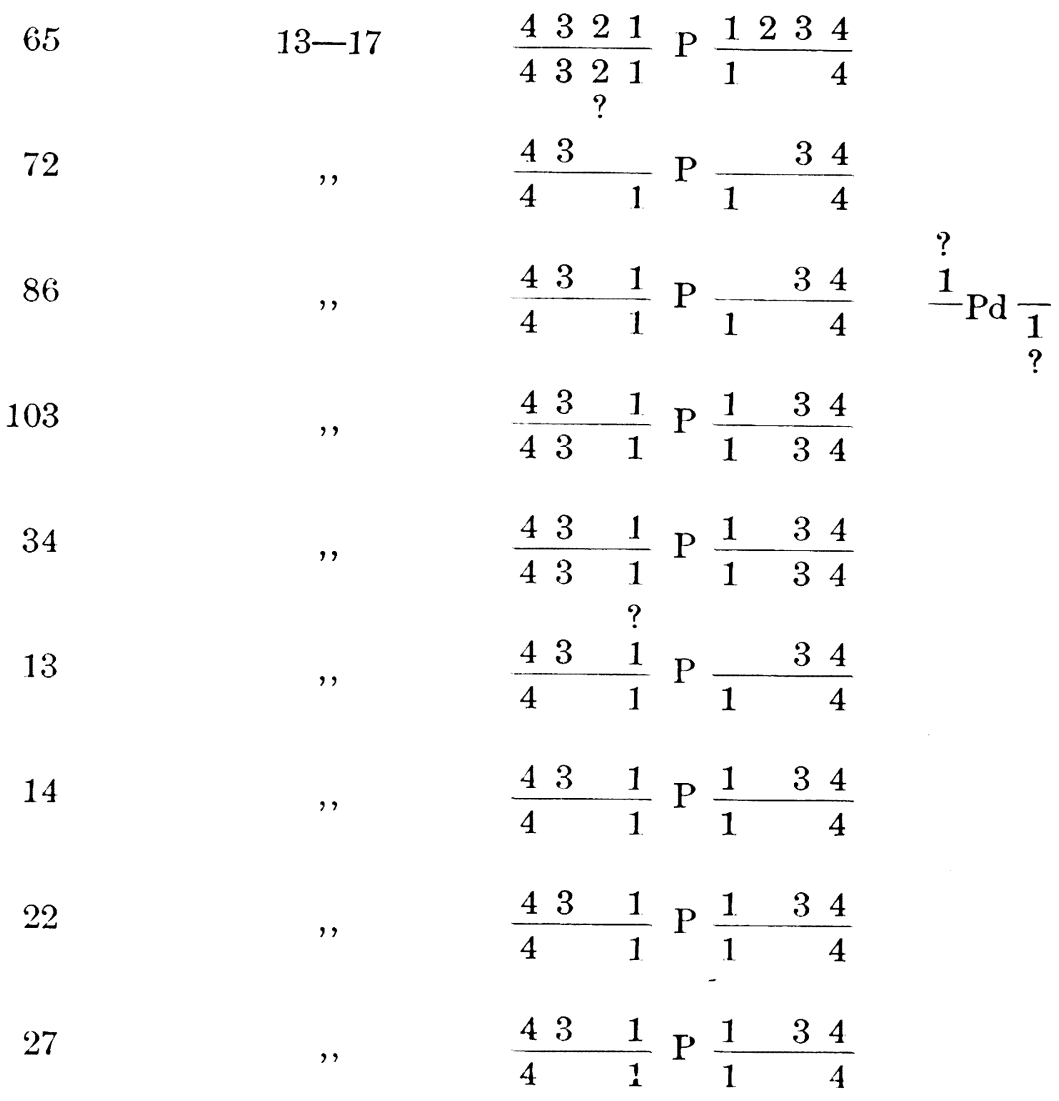


Aus der Tabelle ergibt sich, dass P4 von konstantem Vorhandensein ist, wie wir bereits wiederholt bemerkt haben. Dagegen kommt P3 manchmal nicht zum Vorschein, und P2 wird noch häufiger vermisst. Es gibt allerdings auch Exemplare, bei denen alle Praemolaren vollständig entwickelt sind (Nr. 47, 50, 51, 57). Also die von Pohle ('23) gegebene Gebissformel für die Eisbären, nämlich :

$$
\mathrm{I} \frac{1 \cdot 2 \cdot 3}{1 \cdot 2 \cdot 3} \mathrm{C} \frac{1}{1} \mathrm{P} \frac{1 \cdot 3 \cdot 4}{1 \cdot 4} \mathrm{M} \frac{1 \cdot 2}{1 \cdot 2 \cdot 3}=36 \text { gilt nicht für }
$$

den Yesso Bären, und daher dürfen wir wohl mit Recht für den letzteren folgende Formel feststellen :

$$
\mathrm{I} \frac{1 \cdot 2 \cdot 3}{1 \cdot 2 \cdot 3} \mathrm{C} \frac{1}{1} \mathrm{P} \frac{1 \cdot 2 \cdot 3 \cdot 4}{1 \cdot 2 \cdot 3 \cdot 4} \mathrm{M} \frac{1 \cdot 2}{1 \cdot 2 \cdot 3}=42 .
$$

\section{Literatur.}

Ehrenberg, K. ('22): Akad. Anz. d. Akad. d. Wiss. Wien. 12.

Kormos, T. ('16): Mitt. a. d. Jahrb. d. Ung. Geol. Reichsanstalt. 23.

Middendorff, A. v. ('53): Sibirische Reise. 2.

Mottl, M. ('34): Vorgetragen in der Fachsitzung d. Ung. Geol. Ges. am 21. Febér. 1934 und Geol. Mitt. d. Ung. Geol Ges.

Pohle, H. ('23): Zool. Anz. 56.

Schäff, E. ('89): Arch. f. Naturg. 1.

Schlosser, M. ('09): Abh. Bayr. Akad. d. Wiss. 2. Kl. 24.

\section{Erklärung der Tafelfiguren (Tafel XI).}

Fig. 1. Schädel Nr. 60. etwa 5 Monate alt. ( $(\mathrm{Pd} 2)$

Fig. 2. Schädel Nr. 74. etwa 6 Monate alt. $(\overline{\mathrm{Pd} 2}, \mathrm{Pd} 3)$

Fig. 3. Schädel Nr. 80. etwa 7 Monate alt.

Fig. 4. Schädel Nr. 48. etwa 8 Monate alt. (P], P3)

Fig. 5. Schädel Nr. 2. etwa 10 Monate alt. (II3, $\underline{\text { P1, P3) }}$

Fig. 6. Schädel Nr. 98. etwa 11 Monate alt. (P1)

Fig. 7. Schädel Nr. 99. etwa 12 Monate alt.

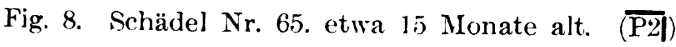

Alle Figuren sind ca. 1/5 nat. (irösse. Die Zähne in der Klammern sind bei der Präparation verloren gegangen. 


\section{Abstract of \\ Über den Zahnwechsel des Yesso Braumbären. Ursus arctos yesoensis Lyd.}

(On the shedding of the milk-teeth of the Yezo brown bear).

\section{By}

Tetsuo Inukai and Kôzô Mukasa

An almost complete series of skulls of the bear native to Hokkaido from the age of 4 months (foetus) to 13 months, at which the permanent dentition is established, and also 4 living young were observed. The first milk-teeth are Id1 and Pd2. The dental formula for the total milk-teeth is $\operatorname{Id} \frac{1 \cdot 2 \cdot 3}{1 \cdot 2 \cdot 3} \operatorname{Cd} \frac{1}{1} \operatorname{Pd} \frac{1 \cdot 2 \cdot 3}{1 \cdot 2 \cdot 3}=28$. Id 1 falls first at the 2 nd month followed by $\mathrm{Pd} 3$ at the 4th, Id2 at the 5th, Id3 at the 7th and $\mathrm{Cd}$ at the 12th month, respectively. The time of disappearing of Pd1 and $\mathrm{Pd} 2$ varies according to the individual. The permanent tooth $\mathrm{P} 1$ or M1 grows first in the one month old young which still keeps all the milk-teeth, and then I1 at the 2nd, I2 at the 3rd, P4 at the 3rd, I3 and M2 at the 4th, M3 at the 5th and $\mathrm{C}$ at the 8 th month, respectively. The premolars P2 and P3 are sometimes lacking, appearing, if at all, after 6 months of age. The original number of premolars was determined as 4 . The dental formula for the bear in question should be $I \frac{\mathbf{1 \cdot 2 \cdot 3}}{\mathbf{1 \cdot 2 \cdot 3}}$ C $\frac{1}{1} \mathrm{P} \frac{1 \cdot 2 \cdot 3 \cdot 4}{1 \cdot 2 \cdot 3 \cdot 4} \mathrm{M} \frac{1 \cdot 2}{1 \cdot 2 \cdot 3}=42$ instead of $\mathrm{P} \frac{1 \cdot 3 \cdot 4}{1 \cdot 4}$ which was given by Pohle ('23) for the polar bear. 


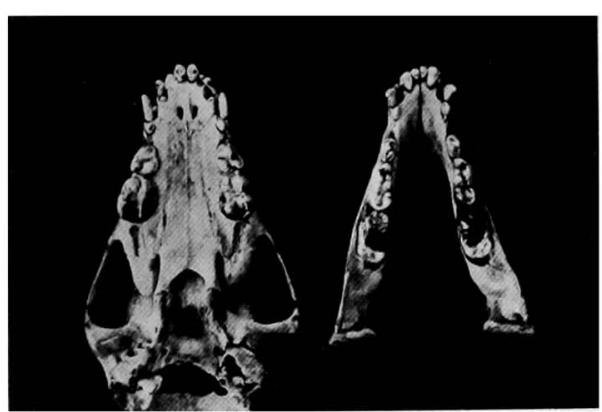

1.

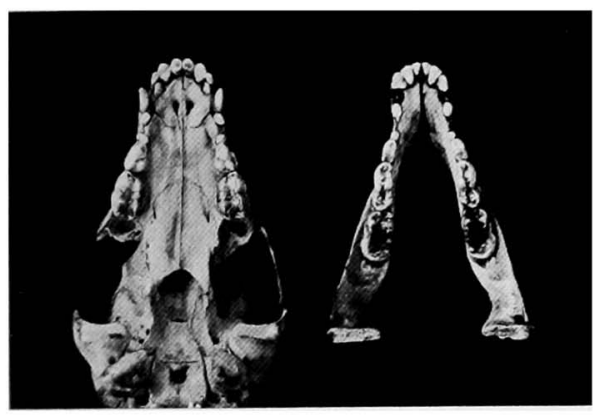

3.

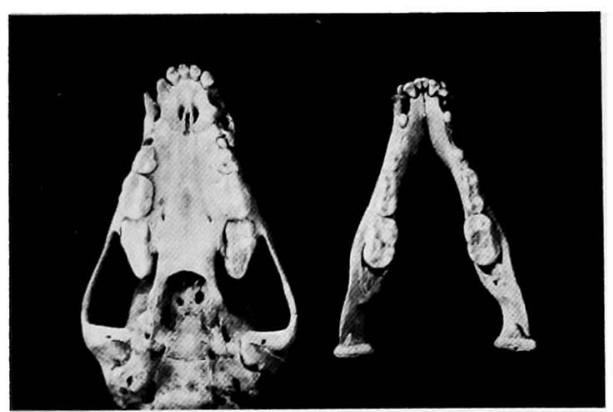

5.

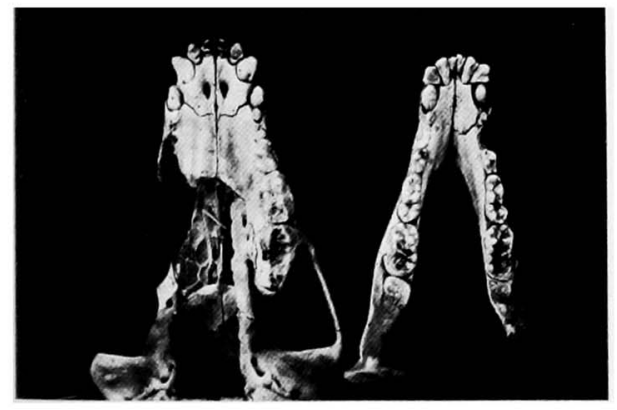

7.

T. Inukai u. K. Mukasa photo.

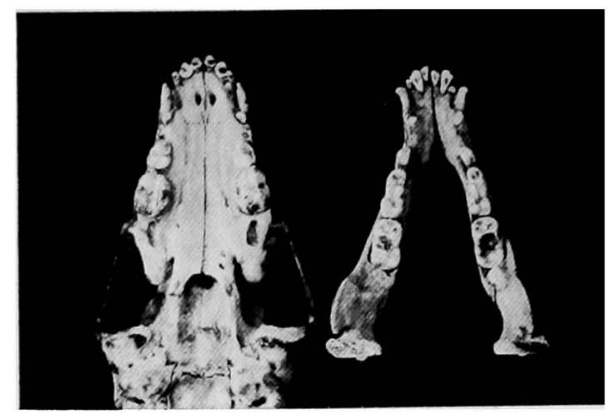

2.

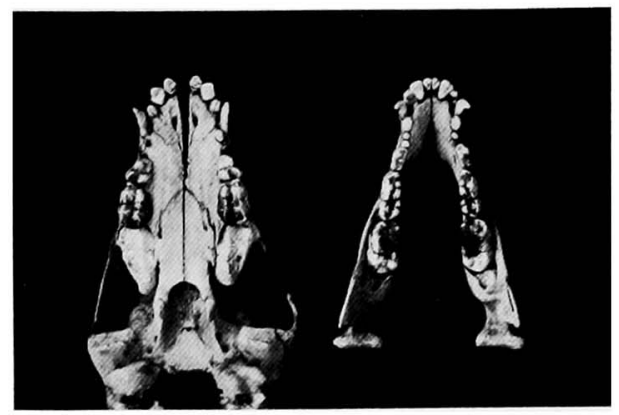

4.

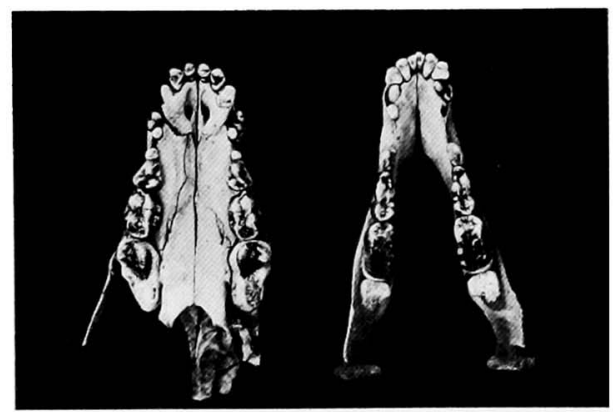

6.

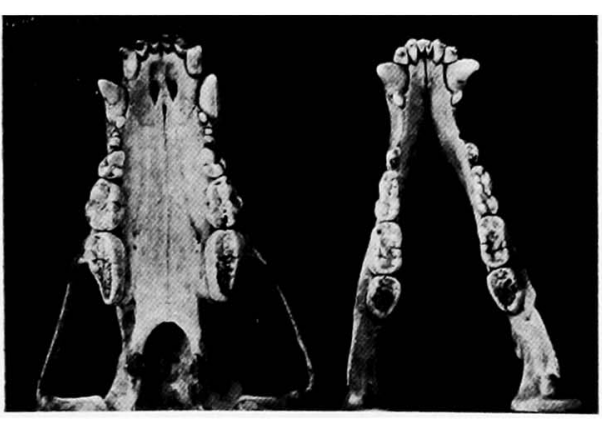

8. 